\section{Questión}

Periodismo / Comunicación ISSN 1669-6581
- Av. $44 \mathrm{~N}^{\circ} 676,1^{\circ}$ piso

CP 1900 - La Plata - Argentina

(a) www.perio.unlp.edu.ar/question

¿Sobre los Incidentes en la cuestión universitaria? ¡Pregúntele a los y las estudiantes!

Matías Causa y Bárbara Torti

DOI: https://doi.org/10.24215/16696581e310

\title{
¿Sobre los Incidentes en la cuestión universitaria? ¡Pregúntele a los y las estudiantes!
}

\section{About the Incidents on the college issue? Ask the students!}

Matías Causa / causamd@gmail.com Profesor y licenciado en Ciencias de la Educación por la UNLP. Especialista en Políticas Educativas y Magíster en Ciencias Sociales con Mención en Educación por FLACSO. Estudiante avanzado del Doctorado en Educación de la FaHCE-UNLP. Docente e investigador en la FTS y la FaHCE-UNLP. Actualmente se desempeña como Prosecretario Académico de la FTS-UNLP.

Bárbara Torti / tortibarbarbara@gmail.com

Licenciada en Diseño Gráfico por la UNNOBA. Profesora de Lengua y Literatura. Estudiante avanzada de la Maestría en Educación de la FaHCE-UNLP (tesis en evaluación). Actualmente se desempeña como adscripta graduada a la docencia universitaria en la cátedra Política e Instituciones Educativas del Profesorado en Trabajo Social FTS-UNLP.

Introducción

Si la pandemia de Coronavirus (Covid-19) algo trajo aparejado en el campo de la educación en general, y de la universidad en particular, es la irrupción de la educación virtual. Cuando lo evitable (amortiguar las brechas producto tanto de las preexistentes como de las nuevas desigualdades que llegan con la virtualidad) irrumpe con la brutalidad de lo definitivo, creyéndoselo, resulta imprescindible que nos orientemos hacia la indagación de los sentidos construidos por los y las estudiantes. Estos sentidos refieren y son configurados por uno de los actores universitarios que pueden resultar quienes, en este escenario, más se constituyan en (y para utilizar una de las categorías últimamente tan en boga que nos indica su identificación) "grupos de riesgo", es decir, en un colectivo ampliamente desfavorecido por la coyuntura a la que asistimos. 
Lejos estamos con este trabajo de pretender esbozar un planteamiento demagógico centrado en los y las estudiantes ni de operar una reducción en cuanto a los múltiples actores universitarios que contribuyen a configurar la universidad pública. A lo largo del texto buscamos participar del debate colectivo acerca de la universidad como institución educativa compleja, enfatizando los sentidos estudiantiles acerca de la experiencia universitaria. Con la debida obligación de explicitar al lector/a desde qué lugar pretendemos participar del debate, diremos que hacemos nuestros los términos de la posición recientemente asumida por el Consejo Interuniversitario Nacional (CIN) en la Declaración denominada "Las universidades argentinas frente a la pandemia del COVID-19":

"Las universidades argentinas no renunciamos a nuestro deber de seguir construyendo el futuro de nuestro país con el mejor antídoto para los males del mundo: el derecho social a la educación"

Dado nuestro enfoque centrado en los y las estudiantes, puntualizamos aquí solo algunas dimensiones de dos recorridos investigativos específicos: uno centrado en las motivaciones y expectativas que entran en juego en la elección de universidad, por parte de estudiantes que transitan el último año de la escuela secundaria, en un distrito de la provincia de Buenos Aires (Torti, 2019)i; el otro sobre los sentidos de la experiencia universitaria que construyen los estudiantes del primer año acerca del estudio, el conocimiento, el aprendizaje y la institución en una de las diecisiete facultades de la UNLP (Causa, 2019)ii. Ambas investigaciones se corresponden con los objetos de estudio de nuestras tesis de posgrado en el marco de carreras realizadas en la misma institución de educación superior. Es por esto que, nuestros trabajos, comparten perspectivas teórico-metodológicas sobre el estudio de los y las estudiantes que están, o bien culminando el último año de la escuela secundaria, o bien transitando el primer año de la formación universitaria, tal como desarrollaremos en los siguientes apartados.

Nos preguntamos, entonces: ¿De qué manera nuestras investigaciones pueden significar aportes para pensar acciones y políticas centradas en las trayectorias de los y las estudiantes que recién llegan o que están por llegar a la universidad? ¿Qué expectativas pueden ser tenidas en consideración a futuro para mejorar las experiencias individuales y colectivas en el tránsito por el primer año? ¿Qué necesidades y/o deficiencias del sistema universitario pone en manifiesto la irrupción de una pandemia en las trayectorias estudiantiles? 
Al formularnos estos interrogantes nos proponemos contribuir con esa idea bastante extendida de que el conocimiento científico es hoy, quizás más que nunca, una fuente de información indispensable para analizar los efectos socioeducativos. De esta manera, procuramos considerar las nuevas formas de desigualdad que pueden derivar de la encrucijada a la que nos enfrenta la pandemia y enfatizar algunos aspectos que deberían ser considerados por las políticas. Nos referiremos, entonces, a algunos hallazgos de nuestras investigaciones que pueden orientar las alternativas en clave propositiva, con el objetivo de ayudar a configurar una agenda universitaria urgente, pero también calibrada para el horizonte de mediano y largo plazo.

La producción académica acerca de los y las estudiantes

En relación con los y las estudiantes Guzmán Gómez y Saucedo Ramos (2015) sostienen que el campo de la investigación se encuentra aún en proceso de consolidación. Señalan que durante la década de los ochenta los y las estudiantes constituían un sujeto desconocido para la investigación educativa y que los estudios que primaban eran de tipo cuantitativo, entendiéndolos como parte de la matrícula o de la población estudiantil. Por otro lado, también existían estudios de corte psicológico centrados en los procesos de aprendizaje.

En los años noventa se produjo un incremento gradual en el interés de las investigaciones por las temáticas referidas a los y las estudiantes, así como su ubicación en las instituciones educativas. Si la pregunta central en la orientación de la investigación era: ¿Quiénes son los estudiantes? En los años noventa pasó a ser: ¿Cómo son los estudiantes?

De esa manera se fue configurando una agenda de investigación centrada en los y las estudiantes y su abordaje comenzó a realizarse mediante estudios de corte cualitativo. En los comienzos del siglo XXI se incrementó el número de investigaciones y se empezó a perfilar la conformación de un campo de estudio a través de la diversificación temática y disciplinaria, y de la profundización en las problemáticas tratadas.iii

Así, las investigaciones dieron cuenta de la heterogeneidad y de nuevas maneras de "ser estudiante". El ingreso cada vez más frecuente de estudiantes que son primera generación universitaria es solo un indicador de los tantos que citan Guzmán Gómez y Saucedo Ramos a propósito de aquellos rasgos que la investigación fue adquiriendo: el ingreso de estudiantes indígenas a la universidad, los foráneos, los trabajadores; el desdibujamiento simbólico entre 
rurales y urbanos, la permanencia de estudiantes madres en niveles educativos superiores, entre otros.

Respecto a las investigaciones sobre aspectos subjetivos de los y las estudiantes universitarios/as, es recién a partir de la primera década de este siglo que su indagación cobra fuerza y se configura como una tendencia clara en la agenda de la investigación. En nuestro país, los trabajos acerca de la problemática de los estudiantes ingresantes a la universidad se centraron fundamentalmente en el análisis del abandono. En su mayoría estos estudios focalizan su atención en la definición del perfil de quienes abandonan los estudios, siendo más recientes los abordajes en torno a los factores institucionales que actúan como condicionantes de dicho problemaiv.

En un análisis de la producción académica publicada entre el año 2002 y el 2012, centrado en las universidades nacionales de la Argentina, García de Fanelli (2014) destaca que el estudio de los factores que inciden sobre el abandono, la graduación y el rendimiento de los y las estudiantes universitarios/as adquirió un nuevo interés. Sin embargo, una parte importante de los trabajos se centró más en los factores individuales (demográficos, socioeconómicos y académicos), prestando una menor atención a los factores organizacionales en la explicación del fenómeno. Es decir, aunque fueron surgiendo, restan aún en la agenda del campo estudios que centren su atención en las dinámicas institucionales.

Por otro lado, se observa otra gran área temática vinculada al estudio de la universidad que aborda las transformaciones recientes de este nivel educativo desde la perspectiva de sus actores. Se trata de investigaciones que indagan en las experiencias, las trayectorias, los relatos, las formas de apropiación y sentidos que los y las estudiantes construyen y expresan. Sus enfoques son principalmente cualitativos y se proponen aportar a la comprensión de las dimensiones socioculturales, antropológicas y pedagógicas (Bracchi, 2005; Carli, 2012, 2014; Cerezo, 2015; Colabella y Vargas, 2013; Pierella, 2014, 2018). En palabras de Pierella, se trata de:

“...un área de estudios sobre la universidad que revela interpretaciones subjetivas de los actores en el marco de fenómenos históricos colectivos, considerando las discontinuidades y continuidades respecto de experiencias anteriores, los puntos de inflexión y procesos de apertura que allí tienen lugar" (2014:21) 
En esta última gran área de interés se ubican los dos recorridos investigativos que presentaremos en el siguiente apartado, dado que sus enfoques se centran en los sentidos que construyen los y las estudiantes, sentidos que contribuyen a organizar su propia vida social y educativa.

La "universidad esperada" por los y las estudiantes de último año de la escuela secundaria Pensar la universidad hoy requiere, sin dudas, aproximarnos también a cómo construyen sus expectativas los y las futuras ingresantes en torno a la educación superior, para lo cual resulta imprescindible tener en cuenta las experiencias juveniles que se dan en el marco de la escuela secundaria. Estas experiencias actualmente tienen lugar en un escenario que combina el aumento de la cobertura del nivel y el consecuente cambio en la composición social de quienes asisten, con un sistema educativo caracterizado por producir y reproducir, de modo fragmentado, las desigualdades sociales. Por otro lado, no se debe perder de vista el surgimiento de nuevas universidades nacionales en el interior del país que implica también la diversificación de los públicos que conforman la matrícula en el nivel superior.

De este modo, los relatos de los y las estudiantes de último año de la escuela secundaria del interior de la provincia de Buenos Aires, nos invitan a reflexionar y a pensar la universidad y sus dimensiones tomando como punto de partida un imaginario construido desde las experiencias en la escuela secundaria.

Sobre los sentidos de la elección: expectativas, motivaciones e inseguridades Uno de los hallazgos de la investigación que resulta clave señalar en este contexto de aislamiento, distanciamiento social y educación virtual es que, para los y las jóvenes, hablar de elección de universidad significa por sobre todas las cosas la definición de un dónde. Dónde, en qué lugar geográfico y bajo qué niveles de autonomía realizarán sus estudios. Esta autonomía se encontró a su vez delimitada por una cuestión clave: irse o quedarse. Irse de su ciudad natal a cursar una carrera en una universidad a varios kilómetros, o quedarse en la universidad o en un instituto local, conviviendo con sus familias en el hogar actual.

Diversos estudios acerca de las trayectorias estudiantiles hacen referencia a la territorialización, entre ellos Legarralde y otros (2018) sostienen que la vida del estudiante universitario se encuentra compuesta por un ámbito extrauniversitario que contempla la 
experiencia que ofrece vivir en la ciudad y sus diferentes interacciones con el territorio. El desplazamiento cotidiano hacia la facultad, desde la experiencia misma del viaje hasta las reuniones en lugares ajenos a la institución educativa, los diferentes barrios, etc. representan una instancia de aprendizaje en sí mismos. De este modo, estudiar los diferentes itinerarios y densidades de los estudiantes es reconocer la variedad y la complejidad de los desplazamientos, pero también permite reconocer la multiplicidad de actividades intra y extrauniversitarias en las que cada estudiante está inserto y que están fuertemente asociadas a su vida en la facultad.

Estas nuevas experiencias que atraviesan, por sobre todo a los y las estudiantes ingresantes del interior, guardan relación con la búsqueda de la independencia y la autonomía que, aunque no se dé en el plano económico, resultó clave en la discusión e incluso en aquellas situaciones en las que los y las jóvenes se veían "obligados/as" a estudiar y permanecer en la misma ciudad por no contar con la posibilidad de trasladarse. En estos casos fue evidente el descontento y la idea de irse una vez finalizados sus estudios.

Cabe preguntarnos, entonces, qué ocurre en este contexto de aislamiento social obligatorio con la búsqueda por la autonomía y con la motivación por transitar los nuevos espacios que manifestaron los y las estudiantes: ¿De qué manera se resignifican, o no, las experiencias en el territorio virtual? ¿Qué límites u oportunidades presenta en este contexto lo institucional como un aspecto generador de nuevos entornos, prácticas y motivaciones?

Por otro lado, en relación con las inseguridades que manifiestan los y las estudiantes y que conforman también el imaginario en torno a la elección de universidad, puede establecerse una distinción entre las inseguridades propias del ámbito académico y aquellas personales $o$ sociales vinculadas a la vida universitaria lejos de la ciudad natal.

Las inseguridades académicas guardan relación con un temor general a no ser capaces de adaptarse al ritmo institucional y a no comprender los textos de estudio de la carrera elegida. Existe una creencia común de que el nivel superior es una instancia en la que los tiempos y las trayectorias individuales no son tomados en consideración, y que cada quien debe "hacerse cargo" de apropiarse de sus aprendizajes.

Las inseguridades personales o sociales, por su parte, fueron manifestadas por la totalidad de los y las estudiantes de la investigación a raíz de las implicancias que trae pensarse en una institución nueva y desconocida, pero son aún mayores en aquellos/as que desean y/o deben 
trasladarse a otra ciudad. Es decir, se evidencia que los y las jóvenes que planean asistir a una universidad masiva como lo es la Universidad de Buenos Aires, manifiestan inseguridades de índole social en relación con no ser reconocidos/as y a no poder establecer vínculos significativos tanto con docentes como con compañeros/as, en ese sentido se refieren a esta circunstancia como la condición negativa de ser "un número más".

Estas inseguridades coinciden, además, con estudiantes que nunca han vivido un cambio de escuela o una mudanza y que no cuentan con demasiadas experiencias de familiares cercanos que hayan accedido al nivel superior universitario (una importante cantidad de los y las estudiantes estará efectivamente accediendo a la condición de estudiante universitario/a por primera vez en su familia). Sin embargo, el miedo a ser juzgados/as muestra una contracara dado que también aparece en el sentido de oportunidad para algunos/as, más que nada para quienes decidieron trasladarse a otra ciudad y de alguna manera esto también resulta una forma de ponerse a prueba a sí mismos/as.

En relación con las inseguridades planteadas que se corresponden con el plano académico, cabe considerar la dificultad que supone para los y las estudiantes la virtualización de su educación en términos de construcción del oficio de estudiante. En otras palabras, podemos preguntarnos: ¿Qué herramientas se brindan desde las diferentes facultades a los y las ingresantes para saldar la posible ausencia de saberes previos en torno a la educación a distancia, a su vez que se estimula y se favorecen prácticas que colaboren a desarrollar su alfabetización académica?

Además, resulta importante pensar de qué manera las prácticas virtuales y el contacto con compañeros, compañeras y docentes a través de una pantalla, permiten un desarrollo vincular positivo y un reconocimiento necesario para que la experiencia de un primer año en la universidad se corresponda con las expectativas previas. Es posible que las inseguridades de índole social o personal puedan verse "postergadas" por la falta de la presencialidad o bien convertidas en motivaciones dependiendo del compromiso puesto al servicio del encuentro virtual y de las herramientas a disposición tanto de estudiantes como docentes.

Podemos decir que las expectativas de los y las estudiantes, entonces, entremezclan construcciones sociales (familiares e individuales), posibilidades materiales, ideales relacionados a una vida universitaria autónoma y a la manera en que las mismas instituciones de educación superior deberían participar en ella. A su vez, se trata de una elección tomada 
por estudiantes del interior de la provincia de Buenos Aires, lo que pone en juego que esas posibilidades materiales sean en primera medida las que decidan cómo se comienza a construir la decisión de irse o quedarse. Al mismo tiempo, de qué manera el interés por una superación personal y ciertas aspiraciones individuales se conjugan con cuestiones ligadas a querer formar parte de un espacio urbano más extenso y con posibilidades de sociabilidad ampliada que las pequeñas ciudades no brindan.

En contracara, "irse a estudiar" en tiempos de pandemia ubica fuera del mapa la cuestión territorial e institucional que se presentaba como primera motivación entre los y las futuras estudiantes y supone el despliegue de nuevas herramientas y recursos quizás no previstos. Se resignifica el sentido atribuido por los y las jóvenes a la autonomía medida, necesariamente, como un distanciamiento geográfico de sus núcleos familiares, proponiendo otras miradas en torno a los modos de cercanía y de vínculos interpersonales.

La "universidad experimentada" por los y las estudiantes en el primer año de la formación universitaria

Los relatos de los estudiantes constituyen la forma en que experimentan la universidad o dicho en otros términos, la experiencia universitaria estudiantil se presenta como productora de sentidos. Inscripto en este enfoque desarrollamos un trabajo de investigación orientado a reconstruir los sentidos retrospectivos de la experiencia universitaria del primer año de estudios de un conjunto de estudiantes que, al momento de las entrevistas en profundidad y los grupos de discusión, se encontraban cursando el segundo año de una carrera en una unidad académica de la UNLP (Causa, 2019). Algunas preguntas ineludibles con las que proponemos dialogar en este apartado son: ¿Qué contienen los relatos de los estudiantes? ¿Cómo y con qué elementos construyen y articulan la experiencia universitaria en el primer año? ¿Qué nos pueden aportar sus voces para pensar un programa de acción en tiempos de pandemia y virtualización de la enseñanza universitaria?

El primer y principal señalamiento que nos interesa proponer en función de los hallazgos de la investigación es que cualquier lineamiento de política universitaria (tanto durante la urgencia de la pandemia como con mirada de mediano y largo plazo) debería estructurarse considerando que los estudiantes de primer año son actores sumamente activos. Encontramos que los sentidos que construyen sobre la experiencia universitaria durante ese tiempo inicial de los 
estudios evidencian un papel mucho más activo del que ciertos discursos sociales (reproductores de modelos bancarios que conciben a los estudiantes como meros depositarios o espectadores pasivo) podrían suponer en cuanto a su proceso de aprendizaje y a sus modos de vinculación con el conocimiento. Asimismo, en los modos en que transitan la experiencia universitaria los estudiantes no se caracterizan por aquello que Ortega (2008) refirió como meras formas de evasión o simples ejercicio de recurrir a los "atajos", ni tampoco por establecer relaciones de absoluta ajenidad con el conocimiento.

Recuperando la pregunta que se formula Derrida en su libro "Universidad sin condición" (2001) acerca del futuro de la universidad en un mundo que experimenta y ha experimentado profundas mutaciones respecto de aquél en que surgió como institución formadora, nos interesa argumentar en torno a la idea de una "experiencia universitaria sin condición". Con esta noción nos referimos a que para los estudiantes la experiencia universitaria del primer año aparece repleta de cuestionamientos, casi nada de ésta queda al resguardo de profundas interpelaciones que los estudiantes realizan y que se expresan en los sentidos de la experiencia que construyen al habitar la universidad pública.

Preguntarnos por estos cuestionamientos en un contexto en que la experiencia universitaria que imaginaron o bien no ha sucedido, se ha interrumpido o, en el mejor de los casos, se ha virtualizado de manera intempestiva y abrupta parece ser un irrenunciable de cualquier programa de acción o conjunto de políticas que las universidades estén desarrollando o previendo desarrollar.

\section{Principales hallazgos de la experiencia universitaria estudiantil}

Un hallazgo de la investigación que inicialmente nos parece interesante señalar en estas circunstancias tan particulares es que, para los y las estudiantes, el tránsito por la experiencia universitaria del primer año difiere sensiblemente del segundo, en sus relatos hacen hincapié en el problema del abandono interanual del primero al segundo año de la carrera. Señalan, además, el sentido crucial de los primeros exámenes parciales durante ese período y su relación con la decisión de interrumpir la carrera. Sus mayores críticas remiten a la evaluación, a la ausencia de criterios (o al menos a su acceso o disponibilidad) y a las modalidades que asumen los exámenes. 
Por otro lado, en relación con la valoración de la calidad de las clases planificadas e implementadas por sus profesores, sus relatos resultan sumamente críticos cuando perciben que las clases o las formas de dictarlas les resultan "malas o aburridas". Aún más, vinculan la "clase vacía" con las formas de dictado que asumen las propuestas pedagógicas de los profesores: "es importante que en primero te guste la clase, si no se hace difícil después".

Sin duda, estos relatos estudiantiles habilitan interrogantes y ofrecen indicios respecto del quehacer de las universidades, las autoridades y los/as profesores/as (sus prácticas de enseñanza, de organización de los programas, de evaluación y acreditación, entre otras) en el contexto, primero de una pandemia y crisis sanitaria a escala mundial y segundo de la virtualización de la enseñanza universitaria que esta circunstancia trajo consigo. También, en función al análisis de los sentidos estudiantiles de la experiencia universitaria, conviene interpelar las hipótesis que subyacen a las acciones y políticas de inclusión universitaria implementadas o por implementar en las distintas escalas o niveles de intervención: ministeriales, presidencias de las universidades, o bien acciones o políticas que anidan más en el plano institucional de cada facultad, departamento, carrera, etc.

Con matices según las singularidades, una gran mayoría de los y las estudiantes hicieron múltiples referencias a dificultades del orden del contexto social, económico e incluso familiar y personal en tanto elementos que atravesaron su experiencia universitaria del primer año de la carrera. No parece más que una verdad de Perogrullo decir que estas situaciones deben ser consideradas por las universidades ante las circunstancias de la pandemia y los "Incidentes" que traen aparejados, sobre todo para quienes más los están padeciendo, para quienes cuentan con menos recursos para capear las inclemencias.

Quizá menos consabidos resulten los señalamientos estudiantiles del tipo: "los profesores tienen que comprender (comprendernos)", "tienen que ser más sensibles con nosotros", "debemos comprendernos mutuamente", "tienen que saber que nosotros no sabemos todos lo mismo ni venimos de las mismas escuelas secundarias", "que no lo digamos en clase no significa que no tengamos un montón de dudas y dificultades". El eje referido a la sociabilidad estudiantil es otro aspecto de suma importancia en los relatos de los estudiantes, ciertamente los lazos entre pares funcionan como un sostén, especialmente para quienes transitan su primer año de estudios en la universidad. Prestar atención a esta configuración de espacios relacionales nos permitió observar que los estudiantes generan instancias que suponen 
"nuevas posiciones subjetivas" (Carli, 2014:59): grupos de estudio, clases de apoyo, ferias de textos, colectivos artísticos, etc. que, con diverso grado de permanencia, propician experiencias significativas.

¿Qué sucede entonces con todo esto que hace a la experiencia universitaria ante la emergencia de los incidentes que llamamos pandemia/cuarentena/aislamiento/crisis sanitaria mundial? Sin lugar a duda la dimensión de la afectividad en los vínculos pedagógicos, la educación emocional (a la que le solemos prestar menos atención en la formación), junto con la reconfiguración de los lazos y espacios relacionales estudiantiles, planteados en conjunto, abren profundos interrogantes para pensar cómo generar nuevas propuestas que ayuden a construir las mejores opciones pedagógicas.

Ciertamente, pensamos que resulta fundamental la capacidad que pueda exhibir la universidad para autointerrogarse en el sentido de plantearse cómo considerar e incorporar estos aspectos relevantes de la experiencia universitaria estudiantil en la inmediatez, pero también una vez que, al retomar las actividades, nos encontremos cara a cara con quienes, a la postre, han resultado los más desfavorecidos ante la virulencia de esta coyuntura.

Reflexiones finales

En este trabajo nos propusimos avanzar en el debate educativo y universitario suscitado y reactualizado por la situación crítica a la que asistimos por la pandemia del Covid-19. Las opciones pedagógicas que echan mano de las tecnologías y la virtualización de la enseñanza deben incluir el registro de aquello "que pasa" y "les pasa" a los y las estudiantes en tanto actores universitarios protagónicos en los procesos de formación.

A partir de la discusión concreta de nuestras investigaciones, presentamos un primer análisis con el propósito de pensar algunos puntos de partida para intervenir en la inmediatez de la experiencia universitaria. Sin embargo, entendemos que las reflexiones que surgen de esta emergencia deben ser consideradas también cuando esta situación excepcional haya pasado, ya que sería un craso error suponer que será posible volver al aula universitaria tradicional iguales a como nos fuimos.

Para finalizar, insistimos sobre la idea de considerar a los y las estudiantes no solo sujetos de aprendizaje, sino también como miembros activos, reflexivos y críticos de la comunidad universitaria. Enfatizamos que no concurren a la universidad exclusivamente para estudiar, es 
allí donde viven (pasan mucho tiempo de su vida), se relacionan con otros/as (pares, docentes, referentes, etc.) y construyen o reconstruyen sus proyectos de vida. En otras palabras, los y las estudiantes encuentran en el espacio universitario un campo de vitalidad que nos impulsa hacia la búsqueda de procesos de valoración y renovación de los modos de pensar y hacer la enseñanza universitaria.

Referencias bibliográficas

BOLIVAR, A. (2005). El lugar de la ética profesional en la formación universitaria. Revista Mexicana de Investigación Educativa, 24 (10), pp. 93-123.

BRACCHI, C. (2005). "Los "recién llegados" y el intento por convertirse en "herederos". Un estudio socioeducativo sobre los estudiantes". Tesis de Maestría-FLACSO-, Buenos Aires, Argentina.

CARLI, S. (2012). "El estudiante universitario. Hacia una historia del presente de la universidad pública". Buenos Aires: Siglo XXI.

CARLI, S. (Comp.) (2014). Universidad pública y experiencia estudiantil. Historia, política y vida cotidiana. Miño y Dávila. Buenos Aires

CAUSA, M. (2019). Sentidos de la experiencia universitaria y tensiones de la formación: el primer año de estudios en la carrera de la Licenciatura en Trabajo Social de la FTS-UNLP desde la óptica de los profesores y los estudiantes (2019-2020). Tesis de Doctorado en curso. Doctorado en Educación. FaHCE-UNLP.

CEREZO, L. (2015). Universidad: tan cerca y tan lejos. Trayectorias universitarias de jóvenes en situación de vulnerabilidad social (Tesis de Maestría). Facultad Latinoamericana de Ciencias Sociales- Sede Académica Argentina, Buenos Aires, Argentina.

COLABELLA, L; y VARGAS, P. (2013). "La Jauretche". Una universidad popular en la trama del sur del Gran Buenos Aires. Buenos Aires: CLACSO. Disponible en: http://www.biblioteca.clacso.edu.ar/clacso/becas/20131218063624/policybrief.pdf (Consultado 17/4/2020)

Consejo Interuniversitario Nacional (8 de abril 2020). Las universidades argentinas frente a la pandemia del COVID 19. CIN. Disponible en: https://www.cin.edu.ar/las-universidadesargentinas-frente-a-la-pandemia-del-covid-19/?fbclid=IwAR1n2J36oGNGpwEY5nFbzxKUxxmy7cLTKCrCBSS4AVDVQy407h52Hw_j6k (Consultado 08/04/2020). 
DERRIDA, J. (2001). Universidad sin condición, Madrid, Trotta

GARCÍA DE FANELLI, A. (2014). Rendimiento académico y abandono universitario: modelos, resultados y alcances de la producción académica en la Argentina". En: Revista Argentina de Educación Superior, Conocimiento y Difusión, año 6, n.8, pp.1-30

GUZMÁN GÓMEZ, C y SAUCEDO RAMOS C. (2015). Experiencias, vivencias y sentidos en torno a la escuela y a los estudios: Abordajes desde las perspectivas de alumnos y estudiantes. Revista Mexicana de Investigación Educativa, 20 (67) 1019-1054

LEGARRALDE, M.; CONTIGNOLA, M. y MARGUELICHE, J.C. (2018). "Las trayectorias estudiantiles territorializadas. Una mirada desde el ingreso a la universidad". Trayectorias Universitarias, $\quad$ vol. $\quad 4, \quad n^{\circ} 6 . \quad$ Disponible en: https://revistas.unlp.edu.ar/TrayectoriasUniversitarias/article/view/5982

(Consultado 24/04/2020)

ORTEGA, F. (2008). Atajos. Saberes escolares y estrategias de evasión. Miño y Dávila, Buenos Aires.

PIERELLA, M.P. (2014). La autoridad en la universidad. Vínculos y experiencias entre estudiantes, profesores y saberes. Paidós, Buenos Aires.

PIERELLA, M.P. (2018). "El primer año de universidad desde la perspectiva de los profesores. Políticas de recepción, enseñanza y currículum”. En: Espacios en blanco. Serie indagaciones, 28(2), 33-48. Disponible en: http://www.scielo.org.ar/scielo.php?script=sci_arttext\&pid=S151594852018000200004\&lng=es\&tlng=es. (Consultado 20/4/2020)

TORTI, B. (2019) "Si no te vas ahora, después no te vas más" La elección de universidad en estudiantes de escuelas secundarias del interior de la provincia de Buenos Aires. Tesis de Maestría. Maestría en Educación. FaHCE-UNLP.

ZABALZA, M.A. (2002). La enseñanza universitaria. El escenario y sus protagonistas. Madrid: Narcea.

Notas

\footnotetext{
i Tesis en proceso de evaluación. Maestría en Educación. FaHCE-UNLP

ii Tesis en curso. Doctorado en Educación. FaHCE-UNLP 
iii También corresponde señalar la emergencia de los estudios sobre el movimiento estudiantil que han profundizado en el papel de los estudiantes universitarios como sujetos políticos con diversos estilos de participación y modalidades organizativas que, fundamentalmente en los últimos 15 años, se caracterizaron por alzar discursos y realizar manifestaciones críticas a distintas medidas restrictivas implementadas por los gobiernos neoliberales (Buchbinder, Califa y Millan, 2010; OSAL, 2012; Vommaro, 2013; entre otros).

iv Corresponde señalar que, desde la mitad de la década del setenta, en el contexto internacional, los aportes de Vincent Tinto (1975) se consideran relevantes por su abordaje del abandono como tema complejo, atravesado por variables individuales e institucionales. A lo largo de toda su obra Tinto destaca el rol que tiene la institución en esta problemática y lo analiza como otro de los posibles condicionantes. 